\title{
Synthesis of Hydroxylated Macroporous Polymer Beads for Microwave-Assisted Desorption of Nonpolar Volatile Organic Compounds
}

\author{
Mi-Lim Hwang, Young-Seok Lee, Tae-Gyun Kim, ${ }^{\dagger}$ Go-Su Yang,,${ }^{\dagger}$ Tae-Seon Park, ${ }^{\dagger}$ and Youn-Sik Lee* \\ Division of Chemical Engineering, Nanomaterials Processing Research Center, Chonbuk National University, \\ Chonju, Chonbuk 561-756, Korea."E-mail: yosklear@jbnu.ac.kr \\ 'Department of Environmental Engineering, Chonbuk National University, Chonju, Chonbuk 561-756, Korea \\ ${ }^{\star} H$-Plus ENG Ltd. 5F J-Tower, 158-3, Seokchon-dong, Songpa-gu, Seoul 138-844, Korea \\ Received April 14, 2010, Accepted June 21, 2010
}

Key Words: Suspension polymerization, Macroporous polymer bead, Nonpolar VOC, Microwave-assisted desorption, Desorption efficiency

Macroporous polymer beads are cross-linked polymers having dry state porosity, and have been widely used as ionexchange resins, chromatography column packing materials, catalyst supporters, absorbents, etc. In general, they are prepared by suspension polymerization of divinylbenzene and styrene (or any other vinyl monomer and cross-linking agents) in the presence of a pore-forming agent (porogen or diluent). ${ }^{1,2}$

Opperman and Brown reported a microwave-assisted volatile organic compounds (VOCs) recovery system with polymer bead adsorbents, with emphasis on significant advantages over the conventional granulated activated carbon-based systems in terms of cost and energy efficiency, but details on their experimental results were not included. ${ }^{3}$ Nonpolar compounds, including nonpolar polymer beads and nonpolar VOCs, are transparent or semi-transparent to microwaves. ${ }^{4}$ Thus, the efficiency of microwave-assisted desorption of nonpolar VOCs such as benzene, toluene, and xylene from nonpolar adsorbents is not satisfactory, since irradiation with microwaves does not efficiently heat either such adsorbents or VOCs.

One approach toward enhancing the microwave-assisted desorption efficiency of nonpolar VOCs from polymer beads makes use of certain polymer adsorbents that can be hydrated to some extent under certain humidified conditions, and consequently heated enough by microwaves for nonpolar VOCs to be desorbed efficiently. Even though some commercially available polymer adsorbents can be wet under humid or ambient conditions, most of them are too hydrophilic to efficiently adsorb nonpolar VOCs.

As a first step to develop such polymer adsorbents that can adsorb nonpolar VOCs and can also be partially hydrated in certain conditions, we prepared macroporous polymer beads via suspension polymerization of a mixture of divinylbenzene and vinyl acetate in the presence of toluene and isooctane as pore-forming agents. The resulting polymer beads were hydrolyzed in an aqueous $\mathrm{KOH}$ solution to give polymer beads bearing hydroxyl groups. For comparison, nonpolar polymer beads were also prepared via suspension polymerization of a mixture of divinylbenzene and styrene. This paper briefly describes experimental results on the microwave-assisted desorption of benzene as well as some fundamental properties of the polymer beads.

\section{Experimental Section}

Materials. Divinylbenzene (57\%) and poly(vinyl acetate) (Polinol P-17) were purchased from Nippon Steel Co. and OCI (South Korea), respectively. Styrene (99\%), tricalcium phosphate $(98-103 \%)$, and toluene were purchased from DAE JUNG. Benzoyl peroxide (97\%) and 2,2,4-trimenthylpentane $(99+\%)$ were purchased from Alfa Aesar and Sigma-Aldrich, respectively. All the chemicals were used as received.

Synthetic procedure of polymer beads. The macroporous polymer beads were prepared by following reported procedures with minor modifications. ${ }^{5,6}$ Briefly, an organic solution of comonomers (total $60 \mathrm{~mL}$ ), benzoyl peroxide $(0.8 \mathrm{~g})$, and toluene/isooctane $(1 / 3$, total $120 \mathrm{~mL})$ was prepared. The amounts of comonomers used for the preparation of the hydroxylated polymer beads (DA20 and DA40) and nonpolar beads (DS20) are shown in Table 1. The aqueous phase was composed of poly(vinyl alcohol) $(0.8 \mathrm{~g})$, tricalcium phosphate $(1.6 \mathrm{~g})$, and water $(540 \mathrm{~mL})$. The combined oil and aqueous solution mixture was polymerized at $80^{\circ} \mathrm{C}$ for $12 \mathrm{~h}$ under stirring at the speed of $1000 \mathrm{rpm}$. The polymer beads precipitated from the reaction mixture were cleaned with water and subsequently with acetone. The beads bearing vinyl acetate units underwent an alkaline hydrolysis in an aqueous $\mathrm{KOH}$ solution. The polymer beads were cleaned using a surfactant (Koremul-FN-10) and solvents. Finally, the beads were extracted with methylene chloride in a Soxhlet apparatus, followed by washing with methanol and drying at $50^{\circ} \mathrm{C}$ for $12 \mathrm{~h}$. The polymer beads were fractionated using ASTM sieves to obtain beads in the range of 0.3 to 0.8 $\mathrm{mm}$ in diameter.

Measurements. The surface morphology of the polymer beads was observed on a scanning electron microscope (model JEOL

Table 1. Monomer mixture compositions for the suspension polymerizations

\begin{tabular}{cccr}
\hline Comonmer & DS20 & DA20 & DA40 \\
\hline Divinylbenzene & $48 \mathrm{~mL}$ & $48 \mathrm{~mL}$ & $36 \mathrm{~mL}$ \\
Styrene & $12 \mathrm{~mL}$ & $0 \mathrm{~mL}$ & $0 \mathrm{~mL}$ \\
Vinyl acetate & $0 \mathrm{~mL}$ & $12 \mathrm{~mL}$ & $24 \mathrm{~mL}$ \\
\hline
\end{tabular}




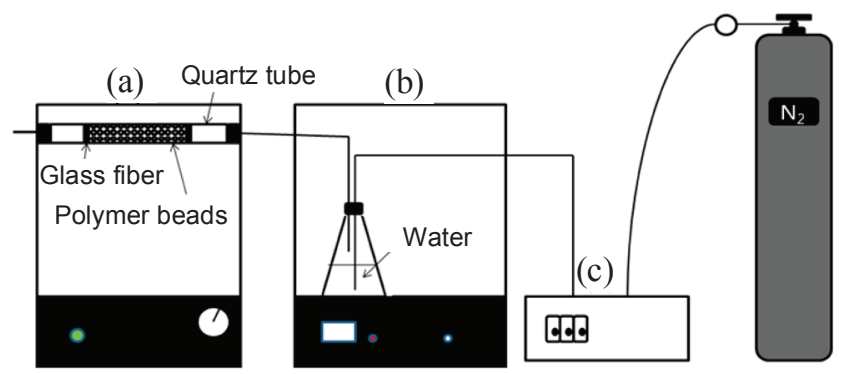

Figure 1. Schematic representation of the microwave-assisted desorption apparatus: (a) a microwave-irradiation chamber, (b) a humidifying oven, and (c) a nitrogen flow rate controller.

FE-SEM-5900) at $20 \mathrm{kV}$ after coating with platinum. The pore size and surface area of the polymer beads were determined by BJH and BET methods, using an ASAP 2010M instrument. ${ }^{7}$ The apparent density and porosity of the polymer beads were determined using a liquid displacement method as described in the literature. ${ }^{8}$ In order to estimate the wetting efficiency of the polymer beads at room temperature, the polymer beads were put into a glass tube, and both ends of the tube were sealed by cotton balls. A humid air stream of $500 \mathrm{~mL} / \mathrm{min}$ (formed through an air-bubbling apparatus at $70-80^{\circ} \mathrm{C}$ ) was supplied into the tube for $30 \mathrm{~min}$, and the polymer beads were weighed. The wetting efficiency for each polymer adsorbent was calculated from the ratio of the weight increase $\left(\mathrm{W}-\mathrm{W}_{\mathrm{o}}\right)$ after wetting to the initial weight $\left(\mathrm{W}_{\mathrm{o}}\right)$ of the beads: wetting efficiency $=[(\mathrm{W}-$ $\left.\left.\mathrm{W}_{\mathrm{o}}\right) / \mathrm{W}_{\mathrm{o}}\right] \times 100$.

Microwave-assisted desorption apparatus. Desorption efficiency of the polymer beads toward benzene was carried out in a home-built apparatus (Fig. 1). In order to calculate \% adsorption of benzene inside the polymer beads, polymer beads $(10 \mathrm{~g})$ dried in an oven at $50^{\circ} \mathrm{C}$ for $10 \mathrm{~h}$ were soaked in $70 \mathrm{~mL}$ benzene for $2 \mathrm{~h}$ at room temperature. Benzene was decanted off, and the remaining polymer beads were dried in a hood at room temperature for $1 \mathrm{~h}$. The $\%$ adsorption was calculated from the ratio of the increase in the bead weight to the initial bead weight. The benzene-adsorbed polymer beads were put into a glass tube $(30 \mathrm{~mm} \times 300 \mathrm{~mm})$, and both ends of the tube were sealed by glass fibers. A nitrogen stream of $500 \mathrm{~mL} / \mathrm{min}$ was supplied into the tube through a water bubbling apparatus (70 $80^{\circ} \mathrm{C}$ ) for humidification of the polymer beads. After treatment the tube with $600-\mathrm{W}$ microwaves for $30 \mathrm{~min}$, the polymer beads were taken off from the tube and weighed, and the desorption efficiency was calculated according to the following equation where $\mathrm{W}_{\mathrm{ad}}$ and $\mathrm{W}_{\mathrm{de}}$ are the weight of benzene adsorbed by polymer beads and the weight of benzene desorbed from the polymer beads during microwave irradiation, respectively: desorption efficiency $(\%)=\left(\mathrm{W}_{\mathrm{de}} / \mathrm{W}_{\mathrm{ad}}\right) \times 100$.

\section{Results and Discussion}

In order to prepare cross-linked polymer bead adsorbents with a near maximum content of hydroxyl group, a mixture consisting of $36 \mathrm{~mL}$ divinylbenzene (in fact, a mixture of 57\% divinylbenzene and $43 \%$ ethylbenzene) and $24 \mathrm{~mL}$ vinyl acetate was chosen ( $40 \mathrm{vol} \%$ in feed), where the pure divinylbenzene content is $34 \mathrm{vol} \%$ ). Since polymer beads prepared using less than $30 \%$ pure divinylbenzene may not be able to maintain their initially formed pores in a dried state because of their relatively lower dimensional stability., ${ }^{9,10}$ Polymer beads with higher contents of vinyl acetate (leading to higher contents of hydroxyl group after hydrolysis), and consequently lower contents of pure divinylbenzene, were not prepared in this study. For comparison, DA20 (vinyl acetate: $20 \mathrm{vol} \%$ in feed) and DS20 (styrene: $20 \mathrm{vol} \%$ in feed) were also prepared using the same procedures. The infrared spectra of the polymer beads before and after alkaline hydrolysis clearly indicated that almost all of the ester carbonyl groups in the vinyl acetate units of DA40 and DA20 were converted to hydroxyl groups.

The apparent density, porosity, BET surface area, and average pore size of the polymer beads in the range of 0.3 to 0.8 $\mathrm{mm}$ in diameter are listed in Table 2. The BET surface area of DA40 was greater than that of DA20, which was greater than that of DS20. In general, the BET surface area of polymer beads increases with increasing divinylbenzene content due to formation of smaller primary particles at higher contents of divinylbenzene. ${ }^{1}$ However, the comonomers used for the preparation of the three different polymer beads were not the same, so this general rule did not apply in the present case. One of the main reasons for the enhanced surface areas of DA20 or DA40 is probably the removal of acetyl groups from the vinyl acetate units in the polymer beads via alkaline hydrolysis, resulting in the formation of additional pores. This hypothesis is experimentally supported by the fact that the BET surface areas of DA40 before and after hydrolysis were estimated to be 544 and $653 \mathrm{~m}^{2} / \mathrm{g}$, respectively. Another important reason for the increased surface areas of DA20 and DA40 seems to be their smaller pores, since the average pore sizes of DS20, DA20, and DA40 were estimated to be $96 \mathrm{~nm}, 87 \mathrm{~nm}$, and $81 \mathrm{~nm}$, respectively. The smaller pores of DA20 and DA40 may be due to the nature of vinyl acetate, which is more polar and consequently more dispersive in the aqueous medium than styrene. The apparent density and porosity of beads are closely related to their BET surface areas and pore sizes. Accordingly, the apparent density of DA40 beads was slightly smaller than that of DA20 beads, which is also slightly smaller than that of DS20, even though their average porosity decreased in going form DS20 to DA40. As expected, SEM micrographs revealed that the surfaces of DA40 beads were significantly more porous

Table 2. Apparent density, porosity, BET surface area, and average pore size of the polymer beads with diameters in a range $0.3-0.8 \mathrm{~mm}$

\begin{tabular}{ccccccc}
\hline Sample Code & Apparent density $(\mathrm{g} / \mathrm{mL})$ & Porosity & Surface area $\left(\mathrm{m}^{2} / \mathrm{g}\right)$ & Average pore size $(\mathrm{nm})$ & Adsorption $(\%)$ & Desorption $(\%)$ \\
\hline DS20 & 0.22 & 0.78 & 424 & 96 & 21 & 39 \\
DA20 & 0.18 & 0.82 & 567 & 87 & 31 & 48 \\
DA40 & 0.14 & 0.88 & 653 & 81 & 40 & 62 \\
\hline
\end{tabular}




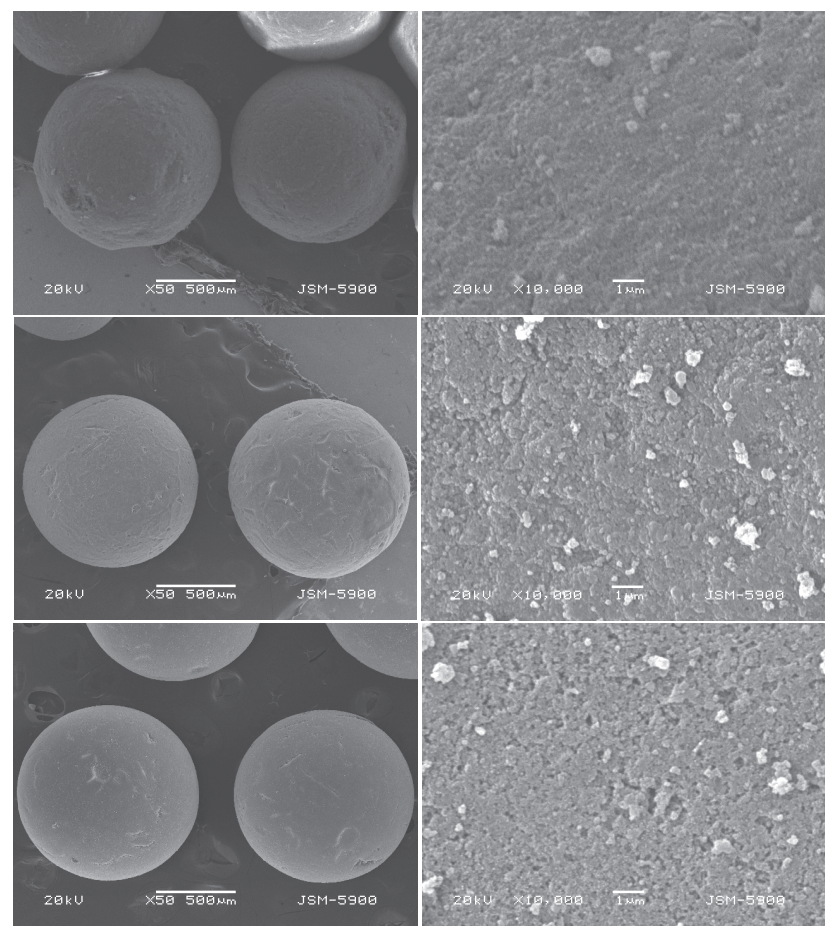

Figure 2. SEM images of the polymer bead shapes and surfaces ( $\times 10,000)$ of DS20 (top), DA20 (middle), and DA40 (bottom).

than that of DS20 beads (Fig. 2).

The amount of adsorbed benzene in DA40 beads $(40 \%)$ was greater than those of DA20 (31\%) and DS20 (21\%), indicating that the hydroxyl groups in the polymer beads do not significantly affect the adsorption of benzene. This result suggests that the BET surface area may be the most important factor affecting benzene adsorption since overall, the DA20 and DA40 beads are relatively nonpolar. The polymer beads swollen with benzene were then exposed in a humid air stream for $30 \mathrm{~min}$ for wetting with water molecules, and treated with $600-\mathrm{W}$ microwaves for $30 \mathrm{~min}$. As expected, the desorption efficiencies of DA40 (64\%) and DA20 (48\%) were higher than that of DS20 (39\%). This result clearly indicates that the incorporation of polar hydroxyl groups into the nonpolar polymer beads enhances desorption efficiency toward nonpolar VOCs. The wetting efficiency of the polymer beads was estimated using the adsorbents without adsorbed benzene under the conditions identical to those for the microwave-assisted desorption process. The wetting efficiency of the polymer beads was calculated by comparing the weights of the beads before and after treatment with the humid air stream. The wetting efficiencies of DS20, DA20, and DA40 were measured to be $21 \%, 28 \%$, and $38 \%$, respectively. This result confirms that the wetting efficiency of the polymer beads can be significantly increased by incorporating hydroxyl groups in the beads.

When the adsorbed water molecules were heated by microwaves, they desorbed from the adsorbents, also increasing the adsorbent temperature. In order to detect any changes in the sample temperatures, the temperatures of DS20, DA20, and DA40 were measured by an optical fiber thermometer (Williamson Co.), and the results of which are shown in Fig. 3. The

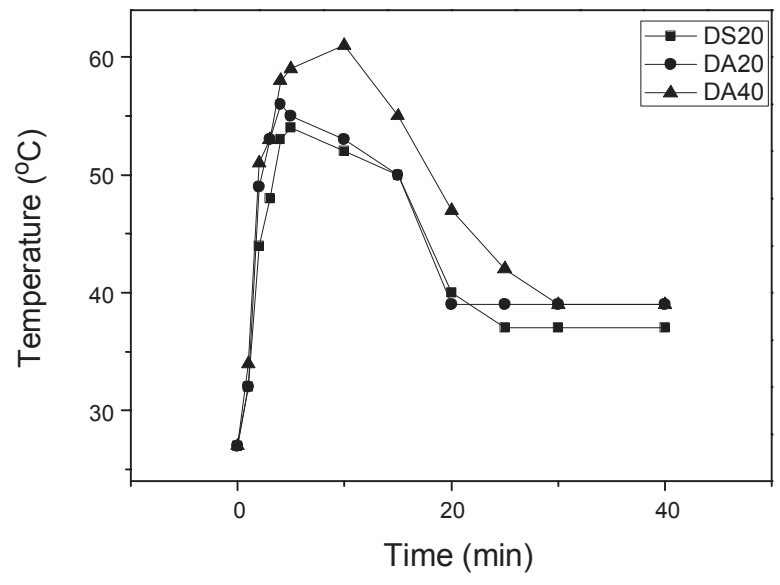

Figure 3. Temperature changes in polymer adsorbents of DS20, DA20, and DA40 in the home-built apparatus during desorption of benzene under $600-\mathrm{W}$ microwave irradiation.

temperature of DA40 increased rapidly up to $61{ }^{\circ} \mathrm{C}$ after $10 \mathrm{~min}$ irradiation with the microwaves, and then decreased slowly to about $38^{\circ} \mathrm{C}$ within $30 \mathrm{~min}$. On the other hand, the DS20 beads were heated up to $56^{\circ} \mathrm{C}$ during the same time period of the irradiation, and more rapidly cooled down to $36^{\circ} \mathrm{C}$ within 25 min. Overall, the temperature change curve of DA20 beads during the microwave irradiation lies between DS20 and DA40, probably because the wetting efficiency of DA20 $(21 \%)$ was between DS20 (28\%) and DA40 (38\%). This result indicates that the initial temperature increase of the polymer beads is primarily due to the adsorbed water molecules on the polymer beads, and depends on the wetting efficiency of the polymer beads.

On the other hand, when the polymer beads were irradiated with the microwaves under non-humidified conditions, all of the three different beads were slowly heated up to $35-38{ }^{\circ} \mathrm{C}$ and then remained constant after about $25 \mathrm{~min}$. This observation suggests that the adsorbed water molecules were almost completely removed from the polymer beads under the conditions, and any effect of the absorbed water on calculation of the desorption efficiency can be ignored. These results indicate that the enhanced desorption of benzene from DA40 is probably due to the more increase in the sample temperature during irradiation with microwaves, as compared to desorption from DS20 and DA20.

In conclusion, macroporous polymer beads containing hydroxyl groups were successfully synthesized, and exhibited greatly improved microwave-assisted desorption efficiency toward benzene, as compared to the nonpolar beads. The enhanced desorption efficiency was primarily attributed to the increased sample temperature caused by the adsorbed water molecules heated by the microwaves. To our best knowledge, this paper is the first report that describes the relationship between polymer adsorbents with well-defined chemical structures and microwave-assisted desorption of a nonpolar VOC. Consequently, we expect that our experimental result can stimulate further development of polymer adsorbents for microwaveassisted desorption of VOCs. 
Acknowledgments. This research was financially supported by Korea Environmental Industry \& Technology Institute (Grant \# 012-071-049).

\section{References}

1. Okay, O. Sci. Prog. Polym. 2000, 25, 711.

2. Seidl, J.; Malinski, J.; Dusek, K.; Heitz, W. Adv. Polym. Sci. 1967, 5, 113

3. Opperman, S. H.; Brown, R. C. Pollution. Eng. 1999, 31, 58.
4. Price, D. W.; Schmidt, P. S. J. Air \& Waste Manage Association 1998, 48, 1146

5. Gupta, D. C.; Beldar, A. G.; Tank, R. J. Polym. Sci. 2006, 10, 3559.

6. Kim, S.-E. Korea patent, 1998, 10-1995-002050.

7. Brunaur, S.; Emmett, P.; Teller, E. J. Am. Chem. Soc. 1938, 60, 309.

8. Ramay, H. R.; Zhang, M. Biomaterials 2003, 24, 3293.

9. Doh, S.-H.; Choi, S.-W.; Kim, J.-B.; Park, S.-W.; Kim, Y.-K. J. Environ. Sci. 2006, 15, 341.

10. Kim, Y.-M.; Ihm, S.-K.; Kim, J.-C.; Lee, D.-K.; Ahn, J.-H. J. Korean Ind. \& Eng. Chem. 1996, 7, 843. 